\title{
Topological-Sector Fluctuations and Curie-Law Crossover in Spin Ice
}

\author{
L. D. C. Jaubert, ${ }^{1,2,3,4, *}$ M. J. Harris, ${ }^{5}$ T. Fennell, ${ }^{6}$ R. G. Melko, ${ }^{7,8}$ S. T. Bramwell, ${ }^{9}$ and P. C. W. Holdsworth ${ }^{1}$ \\ ${ }^{1}$ Laboratoire de Physique, École Normale Supérieure de Lyon, Université de Lyon, CNRS, \\ 46 Allée d'Italie, 69364 Lyon Cedex 07, France \\ ${ }^{2}$ Max-Planck-Institut für Physik komplexer Systeme, 01187 Dresden, Germany \\ ${ }^{3}$ Theoretical Physics, Oxford University, Oxford, OX1 3NP, United Kingdom \\ ${ }^{4}$ OIST_Okinawa Institute of Science and Technology, Onna-son, Okinawa 904-0495, Japan \\ ${ }^{5}$ School of Divinity, University of Edinburgh, New College, Mound Place, Edinburgh, EH1 2LX, United Kingdom \\ ${ }^{6}$ Paul Scherrer Institut, 5232 Villigen PSI, Switzerland \\ ${ }^{7}$ Department of Physics and Astronomy, University of Waterloo, Waterloo, Ontario N2L 3G1, Canada \\ ${ }^{8}$ Perimeter Institute for Theoretical Physics, Waterloo, Ontario N2L 2Y5, Canada \\ ${ }^{9}$ London Centre for Nanotechnology and Department of Physics and Astronomy, University College London, 17-19 Gordon Street, \\ London WC1H OAH, United Kingdom \\ (Received 27 April 2012; revised manuscript received 13 September 2012; published 21 February 2013)
}

At low temperatures, a spin ice enters a Coulomb phase-a state with algebraic correlations and topologically constrained spin configurations. We show how analytical and numerical approaches for model spin-ice systems reveal a crossover between two Curie laws. One of these laws characterizes the hightemperature paramagnetic regime, while the other, which we call the "spin-liquid Curie law," characterizes the low-temperature Coulomb-phase regime, which provides implicit evidence that the topological sector fluctuates. We compare our theory with experiment for $\mathrm{Ho}_{2} \mathrm{Ti}_{2} \mathrm{O}_{7}$, where this process leads to a nonstandard temperature evolution of the bulk susceptibility and the wave-vector-dependent magnetic susceptibility, as measured by neutron scattering. Theory and experiment agree for bulk quantities and at large scattering wave vectors, but differences at small wave vectors indicate that the classical spin-ice states are not equally populated at low temperatures. More generally, the crossover appears to be a generic property of the emergent gauge field for a classical spin liquid, and it sheds light on the experimental difficulty of measuring a precise Curie-Weiss temperature in frustrated materials. The susceptibility at finite wave vectors is shown to be a local probe of fluctuations among topological sectors on varying length scales.

DOI: 10.1103/PhysRevX.3.011014 Subject Areas: Magnetism, Statistical Physics, Strongly Correlated Materials

\section{INTRODUCTION}

There are indications [1-10] that condensed matter physics is leaving the drought in which experimental candidates for quantum spin-liquid states are scarce. Main contenders for future spin-liquid materials are quantum versions of the well-known "spin ices," where quantum fluctuations [11-14] of the classically degenerate spin-ice state could lead to a collective paramagnetic phase with such exotic features as emergent gauge symmetry or charge or spin fractionalization $[15,16]$. Theoretical investigations have had great success in characterizing quantum spin liquids through emergent topological properties [17]. In the case of gapped spin liquids, the presence of topological invariants naturally leads to the concept of "topological order" - $\mathrm{a}$ quantity connected to the topological degeneracy of the emergent gauge theory. Such topological invariants are, however, generally hidden from conventional probes and

\footnotetext{
*ludovic.jaubert@oist.jp
}

Published by the American Physical Society under the terms of the Creative Commons Attribution 3.0 License. Further distribution of this work must maintain attribution to the author(s) and the published article's title, journal citation, and DOI. typically require measurement of nonlocal quantities, such as the entanglement entropy $[18,19]$, which are not obviously accessible to experiment. In the case of gapless spin liquids, entanglement entropy cannot be so easily used to classify the emergent topology. This is particularly unfortunate for the quantum spin ices, which, due to the nature of the underlying classical gauge structure, most likely fall into this category [3]. For this reason, it is imperative to search for other probes that can be used to characterize the topological structure of spin-ice states-probes that are preferably amenable to experimental measurement.

Topological invariants have already been used extensively in the past to characterize the rich behavior of classical, geometrically frustrated magnets and dimer systems, arising from the constraints inherent in these many-body systems. The most familiar topological invariant is the "winding number sector," which can be used to define $Z_{2}$ or integer topological sectors in a variety of $2 \mathrm{D}$ and $3 \mathrm{D}$ systems. Interestingly, other novel topological sectors have recently been identified [20] that are unique to three dimensions and which may be related to exotic quasiparticle excitations in some spin-liquid phases arising from local constraints [21]. In this paper, we address the question of how these topological structures could be related to 
measurable correlations in real materials. The correlations induced by local constraints were discussed in systems on the kagome lattice with both discrete [22] and continuous symmetry [23] and for frustrated antiferromagnets with a pyrochlore structure [24-26]. In these theoretical models, the physics of the "Coulomb phase," with dipolar correlations showing up as pinch-point singularities in reciprocal space [27-29], is universally present. Among systems showing Coulomb-phase physics [30], spin-ice models [31] have again proven particularly fruitful testing grounds for the collective behavior associated with topological constraints, as the associated real materials are the only experimental magnetic systems for which sharp pinch points have been observed [28,29,32,33]. An extensive manifold of low-energy states can be constructed by ensuring that the local constraint - the ice rule-is obeyed on every tetrahedron of the underlying pyrochlore lattice. The ice rule requires that, at low temperatures, two spins point into and two spins point out of every tetrahedron, as shown in Fig. 1. This local divergence-free condition is sufficient to

(a)

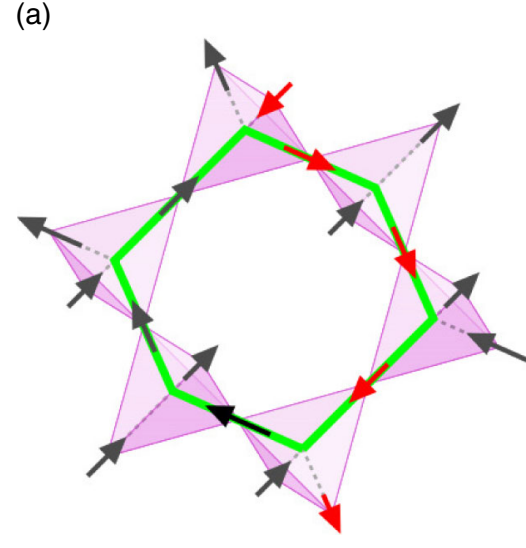

(b)

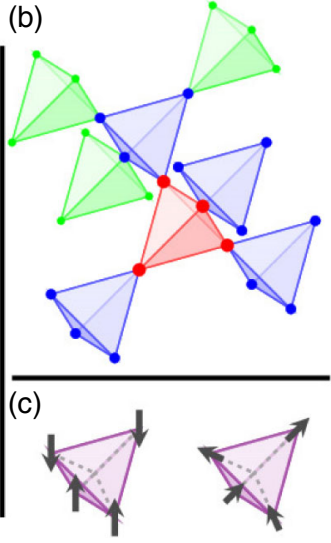

(d)

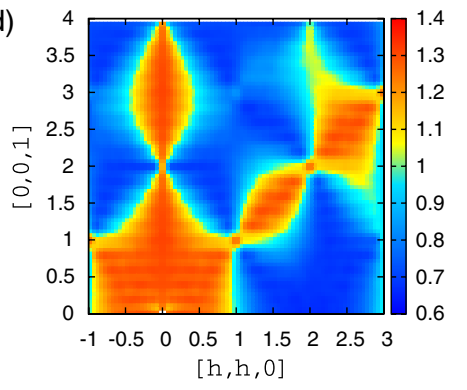

FIG. 1. (a) A portion of the pyrochlore lattice, made of cornersharing tetrahedra. All spins respect the ice rules (two in, two out). The red spins form a string propagating along the $-\hat{z}$ axis, while the green hexagon represents a nonwinding loop of six spins. (b) The Husimi-cactus construction for the pyrochlore lattice. (c) Mapping between spins with easy-axis anisotropy respecting the symmetry constraints of the lattice (right), and the corresponding pseudospins (left). (d) Scattering function $S(\mathbf{Q}, T)$ of the NNSI model at $T=1 \mathrm{~K}$ for a system of 4000 spins, simulating $\mathrm{Ho}_{2} \mathrm{Ti}_{2} \mathrm{O}_{7}$ with $J_{\text {eff }}=1.8 \mathrm{~K}$. The pinch points are evident at $[0,0,2],[1,1,1]$, and $[2,2,2]$. generate the long-range dipolar correlations of the Coulomb phase, even within the confines of the simplest model with nearest-neighbor spin interactions. Fitting the correlated spin configurations into a space with particular boundary conditions leads to the identification of a topological invariant, the winding number, which is directly related to the magnetization (see Sec. II). Hence, although the topological invariants are global measures of the system, they are intimately related to correlations at the microscopic level. It is therefore reasonable to suppose that, by probing the correlations, one can extract information about the invariants themselves. This is the subject of the present paper.

We show that, while spin ice is topologically constrained, it is not topologically ordered, as it is able to fluctuate between topological sectors. We hence show how the susceptibility can be used as an indicator of these topologicalsector fluctuations (TSF) and present a detailed comparison of our results with both bulk-magnetometry and neutronscattering measurements on $\mathrm{Ho}_{2} \mathrm{Ti}_{2} \mathrm{O}_{7}$ [34]. We compare the experimental data to analytical and numerical expressions of the susceptibility, where the emergence of TSF at low temperatures appears as a crossover between two Curie laws at specific wave vectors $\mathbf{Q}$ in the structure factor $S(\mathbf{Q})$. This study was motivated, in part, by the experimental observation of an unusual temperature dependence in the wavevector-dependent susceptibility, close to the Brillouin-zone boundary [35] (see Fig. 6), which is explained by the theory presented in this paper. Approaching the zone center, the experimental data remain consistent with topological-sector fluctuations over all length scales, although there is some discrepancy between theory and experiment, which we discuss.

The rest of the paper is organized as follows: In Sec. II, we present a detailed theoretical discussion of topologicalsector fluctuations in spin-ice models and materials. In Sec. III, we present a calculation of the susceptibility and pseudospin susceptibility within the Husimi-cactus approximation for spin ice, which can be seen as an infinite-dimension version of the pyrochlore lattice. In Sec. IV, we compare theory and experimental data for bulk-magnetometry measurements, both in the context of topological-sector fluctuations and in the extraction of an effective Curie-Weiss temperature. In Sec. V, we compare the numerical simulation of neutron-scattering intensities for nearest-neighbor spin ice with the theory from the Husimi-cactus calculation. Section VI compares theory and neutron-scattering data. In Sec. VII, we broaden the field, showing how our results apply generally to frustrated ferromagnets and antiferromagnets on pyrochlore and related lattices, and we conclude in Sec. VIII.

\section{TOPOLOGICAL SECTORS IN SPIN ICE}

Let us first characterize a model system, of size $L$, with periodic boundaries and, only afterwards, relate its properties to experimental observables. We study the 
nearest-neighbor spin-ice model (NNSI) [31] with vector spins of unit length, $\vec{S}_{i}$, placed on the vertices of a pyrochlore lattice. The lattice is constrained to lie along the body- centered crystal-field directions of the tetrahedra: $\vec{S}_{i}= \pm \vec{d}_{i}$ (see Fig. 1). The NNSI also has ferromagneticexchange interaction $J>0$. The model maps onto Anderson's Ising antiferromagnet [36] with exchange constant $J^{\prime}=-J / 3$ [37] through the definition of Ising pseudospin variables $\sigma_{i}=\vec{S}_{i} \cdot \vec{d}_{i}$. However, it is the ferromagnetic spin-ice model that is physically realizable: The Ising pyrochlore antiferromagnet with a single easy axis cannot represent any real magnetic system, as its Hamiltonian breaks the cubic lattice symmetry [31]. The ensemble of ground states satisfying the ice rules of two spins in and two out, or $\sum_{i=1,4} \sigma_{i}=0$, on each tetrahedron is a Coulomb phase $[24,25]$ and leads to the Pauling zeropoint entropy [38,39] (see Fig. 1). In this paper, we consider cubic systems with $L^{3}$ unit cells and $N=16 L^{3}$ spins. Throughout the paper, as we have in mind comparisons with $\mathrm{Ho}_{2} \mathrm{Ti}_{2} \mathrm{O}_{7}$, we take $J=1.8 \mathrm{~K}$, the value found to best parametrize thermodynamic measurements of this spin-ice material with nearest-neighbor coupling [34]. The strong uniaxial crystal fields [40] make the magnetic degrees of freedom Ising-like up to the $100 \mathrm{~K}$ energy scale, as discussed in more detail in Sec. IV B.

A microstate of the Coulomb phase can be classified by a set of strings of alternating out, in, out ... spins that wind through the system along each of the cubic axes. In other words, any spin $\vec{S}_{i}$ with a downward (upward) projection along a given cubic axis always has at least one nearest neighbor above and below it with the same downward (upward) projection (see Fig. 1). Connecting these spins draws a map of strings spanning the system from top to bottom in the negative (positive) direction. We define an individual string, which does not necessarily close on itself, as an object spanning the system once along one cubic axis, so each string is composed of $4 L$ spins. Hence, each microstate has $n_{k}^{-}\left(n_{k}^{+}\right)$strings spanning in the negative (positive) direction along cubic axis $\hat{k}$, such that the total number, $n_{0}=n_{k}^{-}+n_{k}^{+}=4 L^{2}$, equals the number of spins on a plane of the pyrochlore lattice perpendicular to $\hat{k}$. Each spin belongs to three strings threading along $\hat{x}, \hat{y}$, and $\hat{z}$. Two strings threading through a tetrahedron in the same direction $\hat{k}$ are indistinguishable and can, in fact, be mapped onto the world lines of bosons living in the $(d-1)$-dimensional space perpendicular to the string direction [41-43]. One can define a topological sector for each configuration through a winding vector $\vec{w}=$ $\left(w_{k}=n_{k}^{+}-n_{k}^{-}\right)_{k=x, y, z}$, whose components are even integers taking values between $\pm 4 L^{2} . \vec{w}$ therefore constitutes a topological invariant with $U(1)$ symmetry.

Spin fluctuations within the Coulomb phase are nonlocal and limited to the flipping of a closed loop of spins identified in two categories (see Fig. 2). First, a nonwinding

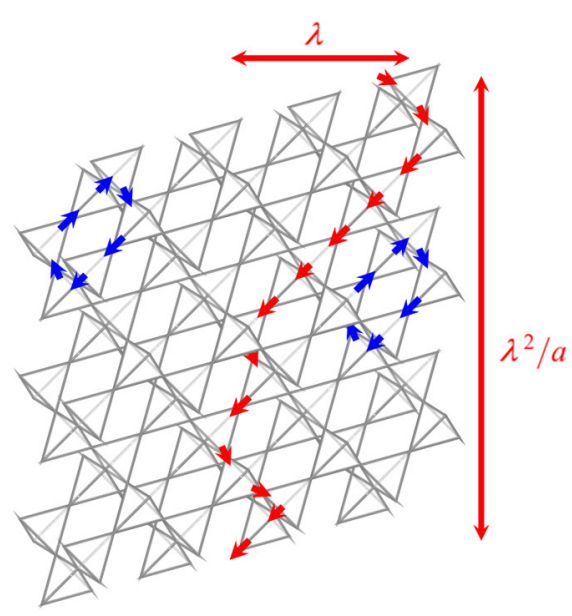

FIG. 2. Nonwinding loop (blue arrows) and string (red arrows) that can be flipped by winding loops of this length. The windingloop move leads to a topological-sector fluctuation. The string (long red arrows) illustrates the effective finite-size system sampled by neutrons, with wave-vector transfer of amplitude $|q|=2 \pi / \lambda$.

loop of spins closes within the system. This kind of spin moves the system between microstates of a given topological sector, rearranging the string network without changing the winding number. Second, a winding loop closes on itself after passing one or more times through the periodic boundaries. Each passage flips a string of spins and changes the topological sector through a change in one component of the winding vector by 2 . We define a topologically ordered system as one that is restricted to a single topological sector.

In model systems, the Coulomb phase space can be sampled directly in simulations by using a nonlocal worm algorithm [44,45]. The worm is a virtual sequence of spins that burrows through the system until it closes on itself, becoming a loop move that maintains the ice rules. Using the worm algorithm, one can test for topological order, or topological-sector fluctuations, without invoking ergodicity arguments. While transitions to topologically ordered states do exist $[28,41-43,47-49]$, no such transition occurs in the NNSI in the zero field, and we find that sector fluctuations are maintained in the Coulomb phase.

Of course, in reality there are no worm dynamics. Rather, magnetic monopoles, which are deconfined topological defects [50,51], provide local dynamics [52], and their creation and annihilation allow for the sampling of different constrained states. Sector fluctuations occur as topological defects are created, wind through the periodic boundaries, and are destroyed. A system-spanning loop update of the worm algorithm is therefore nothing more than a fast forward of such a trajectory for a single pair of topological defects. The same topological defects that provide the dynamics decouple the topological-sector information from the magnetization for length scales above their mean separation. This decoupling happens because 
the magnetization of a lattice plane that cuts a line between the pair of defects is different from one that does not. Hence, strictly speaking, such fluctuations can never be seen in the thermodynamic limit. Pragmatically, there is, of course, a limit for the defect concentration, below which the magnetic fluctuations faithfully represent those of the topological sector. We are able to reach this temperature in simulation and approach it in experiment, allowing us to extract information about fluctuations of the topological sector from the temperature range where the concentration of topological defects is finite.

Topological order may also be defined heuristically in classical spin liquids as a lack of ergodicity between configurations with different topological invariants (see, e.g., Ref. [53]). In this paper, following the discussion above, we show that, in the NNSI with periodic boundaries and in $\mathrm{Ho}_{2} \mathrm{Ti}_{2} \mathrm{O}_{7}$, this heuristic definition of topological order is not required, as on decreasing the temperature the fate of the topological sector fluctuations is decided before ergodicity is lost.

The winding vector $\vec{w}$ is a direct measure of the difference between upward and downward projections along each cubic axis, making it proportional to the magnetic moment: $\vec{M}=(4 L / \sqrt{3}) \vec{w}$. Magnetic fluctuations of a system in the Coulomb phase are therefore a direct measure of topological-sector fluctuations: Nonwinding loop fluctuations carry no magnetic moment, while winding ones carry a magnetic fingerprint of the change in topological sector. Each sector is associated with an extensive subset of states with constant magnetization connected by nonwinding loop moves, while magnetic fluctuations correspond to a change in topological sector. Susceptibility measurements could therefore be used as a diagnostic of both the crossover from the high-temperature paramagnetic phase into the constrained phase and the extent of topological-sector fluctuations at low temperatures.

\section{SPIN ICE ON A HUSIMI CACTUS}

We calculate both the magnetic susceptibility and the pseudospin susceptibility for Ising spins exactly on a Husimi cactus of corner-sharing tetrahedra. This approach preserves the coordinations of the pyrochlore lattice but neglects nonwinding loops (see Fig. 1). Although this approach gives an approximation of the pyrochlore lattice, previous work [41] suggests that it should provide an excellent basis for describing magnetic fluctuations, as it allows system-spanning strings of flipped spins and hence winding-number fluctuations. The approach is reminiscent of the cluster-variation method developed in Ref. [54]. Spins on the $(n+1)$ th shell (green in Fig. 1(b)) have three equivalent neighbors and one neighbor on the $n$th shell (blue in Fig. 1(b)). The total partition function is built up recursively by summing over the degrees of freedom of the spins from the $(n+1)$ th shell while holding the spin on the $n$th shell fixed, with an external field $h$ along the $z$ axis breaking the up-down symmetry $[55,56]$. In order to neglect boundary effects, thermodynamic quantities, such as the longitudinal susceptibility $\chi$, are extracted from the center of the cactus:

$$
\chi \equiv\left(\frac{\partial m_{z}}{\partial h}\right)_{h \rightarrow 0}=\frac{2 \beta}{3} \frac{1+e^{2 \beta J}}{2+e^{2 \beta J}+e^{-6 \beta J}},
$$

where $m_{z}=\left\langle\sum_{i} S_{i}^{z} / N\right\rangle$, and $\langle\cdots\rangle$ is a thermal average. Here and throughout the paper, our susceptibility has dimensions of inverse temperature. To compare with experiment, the susceptibility must be multiplied by $3 C$, where $C$ is the normal Curie constant in the SI units system: $C=\mu_{0} \mu^{2} N_{\mathrm{Ho}} / 3 V \approx 4 \mathrm{~K}$ for $\mathrm{Ho}_{2} \mathrm{Ti}_{2} \mathrm{O}_{7}$, where $\mu_{0}$ is the vacuum permeability, $\mu$ is the magnetic moment operator for $\mathrm{Ho}^{3+}, N_{\mathrm{Ho}}$ is the number of Ho ions, and $V$ is the system volume.

The asymptotic limits of $\chi(T)[44,51]$ reveal a crossover between collective paramagnetic regimes that are unconstrained and constrained, with the Curie constant scaled by a factor of 2:

$$
\chi(T \rightarrow \infty) \sim 1 / 3 T, \quad \chi(T \rightarrow 0) \sim 2 / 3 T .
$$

The factor of $1 / 3$ at high temperatures is a necessary property of a system with cubic space symmetry, familiar in the case of a Heisenberg paramagnet where an applied field couples to fluctuations in only one of the three Cartesian components of the magnetization. We refer to the low-temperature behavior as the "spin-liquid Curie law." Although spin ice has local easy-axis anisotropy, it is isotropic in linear response, like a Heisenberg ferromagnet, as can be seen within the Husimi-cactus calculation. This symmetry is a key property of spin ice and is related to the almost-perfect screening of the long-range interactions in the Coulomb phase of the dipolar spin-ice model [57,58]. Further insight into this low-temperature crossover can be gained from the pseudospin susceptibility (cf. Fig. 1)

$$
\chi_{0} \equiv\left(\frac{\partial m_{0}}{\partial h_{0}}\right)_{h=0}=4 \beta \frac{1+e^{-6 \beta J}}{4+6 e^{2 \beta J}-2 e^{-6 \beta J}},
$$

where $m_{0}=\sum_{i} \sigma_{i} / N$ and where $h_{0}$ is parallel to the global pseudospin axis and thus conjugate to $m_{0}$. The asymptotic limits of $\chi_{0}$ are

$$
\chi_{0}(T \rightarrow \infty) \sim 1 / T, \quad \chi_{0}\left(T \rightarrow 0^{+}\right) \sim \frac{2 e^{-2 \beta J}}{3 T}
$$

without the factor of $1 / 3$, as all pseudospins are parallel to the field. As the constraints are imposed, the pseudospin moment vanishes on each tetrahedron, and $\chi_{0}$ falls to zero on the same temperature scale as the crossover of $\chi$ between the two Curie laws for the system with real spins (cf. Fig. 3), reinforcing our claim that this crossover can be used as a signal of the system entering the Coulomb phase and as an indicator of topological-sector fluctuations at low temperatures. 


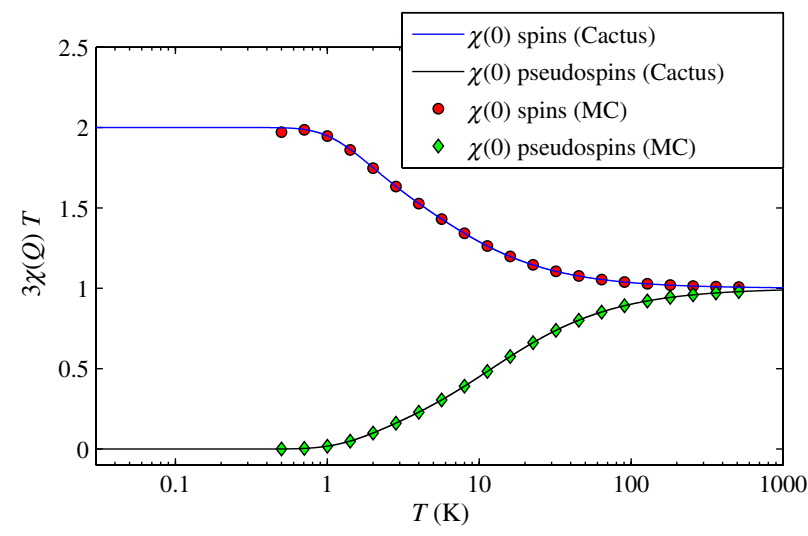

FIG. 3. Analytical and numerical calculations of the spin and pseudospin susceptibilities showing the temperature dependence of $3 \chi T$ for spins, from Eq. (1), and $3 \chi_{0} T$ for pseudospins, from Eq. (3). All data are for $J_{\text {eff }}=1.8 \mathrm{~K}$. The susceptibility of the NNSI model obtained by MC simulation is also compared with the analytical theory.

This result can be attained from fluctuations as well as response, which exposes the origin of the crossover in terms of correlations. The susceptibility can be written as

$$
3 \chi T=1+\sum_{i \neq 0}\left\langle\mathbf{S}_{i} \cdot \mathbf{S}_{0}\right\rangle
$$

where the summation is over the shells of the Husimi cactus. Within the Coulomb phase, the total correlation between $i$ th nearest neighbors is $\left\langle\mathbf{S}_{i} \cdot \mathbf{S}_{0}\right\rangle=2 / 3^{i}[55,56]$, and $\chi=2 / 3 T$ is recovered upon the summation over all shells. We find that most $(98 \%)$ of the crossover is accounted for by the first three shells, i.e., within a sphere of radius $r \lesssim a$. This calculation shows explicitly how integration over all space leads to a masking of the long-range part of the correlations generated by the topological constraints. Hence, we obtain enhanced paramagnetic fluctuations, rather than the critical scattering one might have expected. However, the change of scale as one passes from high to low temperatures is confirmed in simulations with the full connectivity of the pyrochlore lattice (see Sec. V), where the algebraic dipolar correlations are recovered. The factor of 2 between the two regimes appears to be related to the dimensionless stiffness of the Coulomb phase flux field [26].

Comparison with Monte Carlo simulations gives excellent agreement between analytics and numerics, as shown in Fig. 3, where we show both $T \chi$ and $T \chi_{0}$ from simulations of the NNSI using the worm algorithm. The data agree with the analytic prediction, within numerical error, over the entire range of temperature from $100 \mathrm{~K}$ to around $0.7 \mathrm{~K}$, at which point the system enters the asymptotic regime characterized by Eqs. (2) and (4). A closer look at $T \chi$ as $T \rightarrow 0$, taken from Ref. [44], gives an estimate of $T \chi=0.66735 \pm 0.0003$, very close (but not equal) to the $2 / 3$ predicted by the Husimi cactus. The first correction to the Husimi-cactus calculation is a loop fluctuation around a six-spin hexagon. These fluctuations appear at the level of the third shell, whose contribution to the susceptibility is already very small, and are consistent with the Husimi calculation's being an excellent approximation for magnetic fluctuations of the NNSI model.

\section{BULK SUSCEPTIBILITY FOR $\mathrm{Ho}_{2} \mathrm{Ti}_{2} \mathrm{O}_{7}$}

\section{A. Comparison with the Husimi cactus}

We now move from the model to real materials, which have no periodic boundaries, so no winding of loops, but any finite window has strings running through it as in the periodic system. Although boundary effects may change the string statistics [22], the same picture of strings and closed internal loops should hold, motivating a detailed comparison between model systems and experiment. Among the frustrated pyrochlore magnets, $\mathrm{Ho}_{2} \mathrm{Ti}_{2} \mathrm{O}_{7}$ is generally considered one of the best candidates for a spin-ice material. In Fig. 4, we superimpose experimental data from susceptibility measurements for a single crystal of $\mathrm{Ho}_{2} \mathrm{Ti}_{2} \mathrm{O}_{7}$ taken between 3 and $15 \mathrm{~K}$, with data corrected to take demagnetizing fields into account. The experimental moment is scaled to $96 \%$ of its full value of $\mu=10 \mu_{B}$ to get the best fit. Even when taking into account this scale factor (which is close to unity), the agreement between experiment, theory, and simulation is remarkably good, indicating that the bulk susceptibility does indeed approach the TSF regime as the temperature becomes of order $J$. Measurements from a powder sample are compatible with this conclusion [59].

\section{B. Consequences for the Curie-Weiss law}

Unfrustrated ferromagnetic or antiferromagnetic order begins at a temperature scale set by the Curie-Weiss temperature, $\left|\Theta_{\mathrm{CW}}\right|$, estimated from a high-temperature expansion for the magnetic susceptibility:

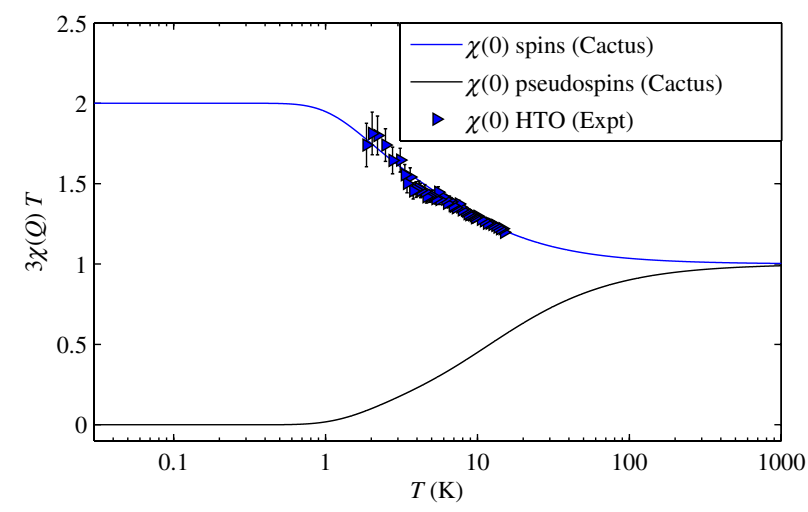

FIG. 4. Solid lines: $3 \chi T$ for spins, from Eq. (1), and $3 \chi_{0} T$ for pseudospins, as in Fig. 3. Blue triangles: Bulk magnetometry measurements of a single crystal of $\mathrm{Ho}_{2} \mathrm{Ti}_{2} \mathrm{O}_{7}$. (See text for details.) 


$$
(3 \chi T)^{-1} \propto 1-\beta \Theta_{\mathrm{CW}} .
$$

Expanding Eq. (1) to order $O(\beta)$, one finds $\Theta_{\mathrm{CW}}=2 J$ for the NNSI. A standard picture of frustrated compounds proposed by Ramirez [60] is that frustration hinders ordering down to a lower temperature, $T^{*} \ll \Theta_{\mathrm{CW}}$, with $T^{*} \rightarrow 0$ for a classical or quantum spin liquid. This is the case for spin ice, although the susceptibility approaches a spinliquid Curie law, characteristic of topological-sector fluctuations, rather than falling to zero at low $T$, as in an antiferromagnetic spin liquid with gapped magnetic excitations. The crossover between the two Curie laws occurs over a very wide range of temperatures so that, while the TSF regime is reached at around $1 \mathrm{~K}$, the paramagnetic Curie law is reached only above $100 \mathrm{~K}$. This requirement to reach rather high temperatures to access the true CurieWeiss law is important for comparison with experiment: $100 \mathrm{~K}$ is outside the temperature range over which $\mathrm{Ho}_{2} \mathrm{Ti}_{2} \mathrm{O}_{7}$ is well approximated by spin-ice models, as the rare-earth single ion $\mathrm{Ho}^{3+}$ starts to lose its Ising nature, becoming more Heisenberg-like at high temperatures $[40,61]$. Although this scale is well above that set by $J$, it is still in the crossover regime. Setting $J=1.8 \mathrm{~K}$, the canonical value $\mathrm{Ho}_{2} \mathrm{Ti}_{2} \mathrm{O}_{7}$ gives $\Theta_{\mathrm{CW}}=3.6 \mathrm{~K}$, which is noticeably larger than the estimate from the bulkmagnetometry measurements for $\mathrm{Ho}_{2} \mathrm{Ti}_{2} \mathrm{O}_{7}$ that are shown in Fig. 5 [34]. In Fig. 5, we plot the data for $1 / \chi$ for the bulk susceptibility against temperature, together with theory and simulation. The data, now scaled by an effective moment that is $96 \%$ of the full moment, compare extremely well with a Curie-Weiss law with $\Theta_{\mathrm{CW}} \sim 1.9 \mathrm{~K}$, but lie far from the true Curie-Weiss law with $\Theta_{\mathrm{CW}}=$ $2 J=3.6 \mathrm{~K}$. In the crossover region, the measured estimate of the Curie-Weiss temperature thus depends on the temperature window used for the fit, leading to an uncertainty in $\Theta_{\mathrm{CW}}$. However, as the frustration parameter is an

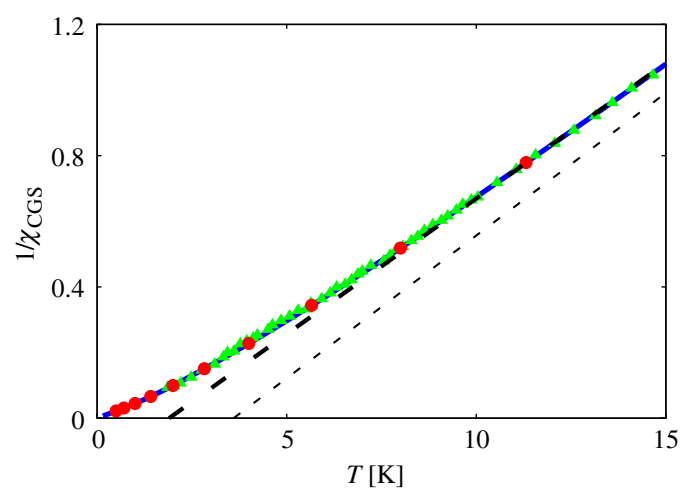

FIG. 5. $1 / \chi$ vs $T$ for bulk-magnetometry measurements of a single crystal of $\mathrm{Ho}_{2} \mathrm{Ti}_{2} \mathrm{O}_{7}$ (green triangles), Monte Carlo simulation of the nearest-neighbor spin ice (red dots), and the analytical result from the Husimi cactus, Eq. (1) (solid blue line). The dashed lines show Curie-Weiss laws for $\Theta_{\mathrm{CW}} \equiv 2 \mathrm{~J}=$ 3.6 K (thin line) and $\Theta_{\mathrm{CW}}=1.9 \mathrm{~K}$ (thick line). The susceptibility is expressed in CGS units $\mathrm{cm}^{3} \mathrm{~mol}^{-1}$. order-of-magnitude concept, this conclusion has only a minor impact on its utilization.

\section{SIMULATIONS OF THE SCATTERING FUNCTION $S(\mathrm{Q}, T)$}

The scattering function $S(\mathbf{Q}, T)$ measured from diffuse unpolarized neutron-scattering intensity in the static approximation is defined as

$$
S(\mathbf{Q}, T)=|f(\mathbf{Q})|^{2}\left\langle\left|\sum_{i=1}^{N} \mathbf{S}_{i \perp} e^{i \mathbf{Q} \cdot \mathbf{r}_{i}}\right|^{2}\right\rangle,
$$

where $\mathbf{S}_{i \perp}$ is the component of a spin at $\mathbf{r}_{i}$ orthogonal to the scattering vector $\mathbf{Q}\left(q_{x}, q_{y}, q_{z}\right)$, and where $\langle\ldots\rangle$ represents a thermal average at temperature $T . f(\mathbf{Q})$ is the magnetic form factor for $\mathrm{Ho}^{3+}$ ions, which we are able to take as unity throughout the paper. $S(\mathbf{Q}, T)$ has previously been evaluated within a mean-field approximation to the dipolar spin-ice model [62]. In this section, we test the generic physics of the Curie-law crossover in the near-neighbor approximation. In the following section, which deals with simulation, the form factor is set to unity, while, when comparing with experimental data, we divide $|f(\mathbf{Q})|^{2}$ out of the problem by scaling the scattering intensity to our theoretical prediction at a fixed high temperature. (See Secs. V and VI). $S(\mathbf{Q}, T)$ is the Fourier transform of the thermally averaged two-spin correlation function, so it is related to the wave-vector-dependent susceptibility.

In the paramagnetic phase, Eq. (7) becomes

$$
S(\mathbf{Q}, T \rightarrow \infty)=\left\langle\sum_{i=1}^{N}\left(S_{i x^{\prime}}^{2}+S_{i, y^{\prime}}^{2}\right)\right\rangle=\frac{2 N}{3},
$$

where $x^{\prime}$ and $y^{\prime}$ are the axes of the plane orthogonal to $\mathbf{Q}$. As a spin ice is cooled into the Coulomb phase, $S(\mathbf{Q}, T)$ develops a strong $\mathbf{Q}$ dependence with, in particular, the appearance of the pinch points $[25,27,63]$ that are characteristic of the local divergence-free constraint imposed by the ice rules. Figure 1 shows a map of $S(\mathbf{Q}, T)$ generated from Eq. (7), in the $[h, h, \ell]$ plane of reciprocal space, for the NNSI as the Coulomb phase is approached. The wave vectors are in units of $2 \pi / a$, where $a$ is the side of a 16-site cubic unit cell. The pinch points, narrow regions of intense diffuse scattering, can be seen at the reciprocal lattice vectors [0,0,2], [1,1,1], and [2,2,2], i.e., at Brillouin-zone centers for the face-centered cubic lattice of the pyrochlore structure. Near the $[0,0,2]$ pinch point, the scattering is expected to take the form

$$
S(\mathbf{Q}, T)=C(T) \frac{\tilde{q}_{z}^{2}+\xi_{\text {ice }}^{-2}(T)}{\tilde{q}_{z}^{2}+\tilde{q}_{x}^{2}+\tilde{q}_{y}^{2}+\xi_{\text {ice }}^{-2}(T)},
$$

where $\tilde{q}_{x}=q_{x}, \tilde{q}_{y}=q_{y}$, and $\tilde{q}_{z}=q_{z}-2 \times(2 \pi / a)$ and where, following Youngblood and Axe [63], $\xi_{\text {ice }}(T)$ is a characteristic length for the Coulomb phase [25,29]. The amplitude $C(T)$, which is the value of $S(\mathbf{Q})$ at the 
singular point $\tilde{q}_{x}=\tilde{q}_{y}=\tilde{q}_{z}=0$, comes from transverse magnetic fluctuations: They are described by spin fluctuations in the plane perpendicular to the wave vector $\tilde{q}_{z}$ that describes them. $S(\mathbf{Q}, T)$ is therefore coupled to two spin components and so should constitute two contributions from the bulk susceptibility $\chi$. This expression predicts a ridge of intense diffuse scattering of constant amplitude along the cubic axis $\left(\tilde{q}_{x}=\tilde{q}_{y}=0\right)$, whose width is limited by $\xi_{\text {ice }}^{-2}$ at the pinch point: By continuity, as $\mathbf{Q}$ goes to zero, $S(\mathbf{Q}=[0,0, \ell], T)=C(T)=2 N T \chi$, which scales as $4 N / 3$ as $T$ goes to zero, while at higher temperatures, as $\xi_{\text {ice }}(T)$ becomes microscopic, the pinch point broadens and one crosses back to isotropic homogeneous paramagnetic scattering, with $S(\mathbf{Q}, T)$ independent of $\mathbf{Q}$ and scaling at around $2 N / 3$. As a summary, we expect

$$
S(\mathbf{Q}, T)=2 N T \chi(T) \begin{cases}\forall \mathbf{Q} & T \rightarrow \infty \\ \forall T & \overrightarrow{\tilde{q}}=\overrightarrow{0} .\end{cases}
$$

In Fig. 6(a), we show simulation results for the NNSI for $3 S(\mathbf{Q}, T) / 2 N$ as a function of temperature for different values of $\mathbf{Q}$. For $\mathbf{Q}$ along [0, 0, $\ell$, the simulation results confirm the above scenario to a high degree of accuracy, as results at the Brillouin-zone boundary $[0,0,1]$ and center $[0,0,2]$ follow the theoretical expression given by the cactus calculation within numerical precision between $T=$ $0.7 \mathrm{~K}$ and $T=1000 \mathrm{~K}$. This agreement should also be true
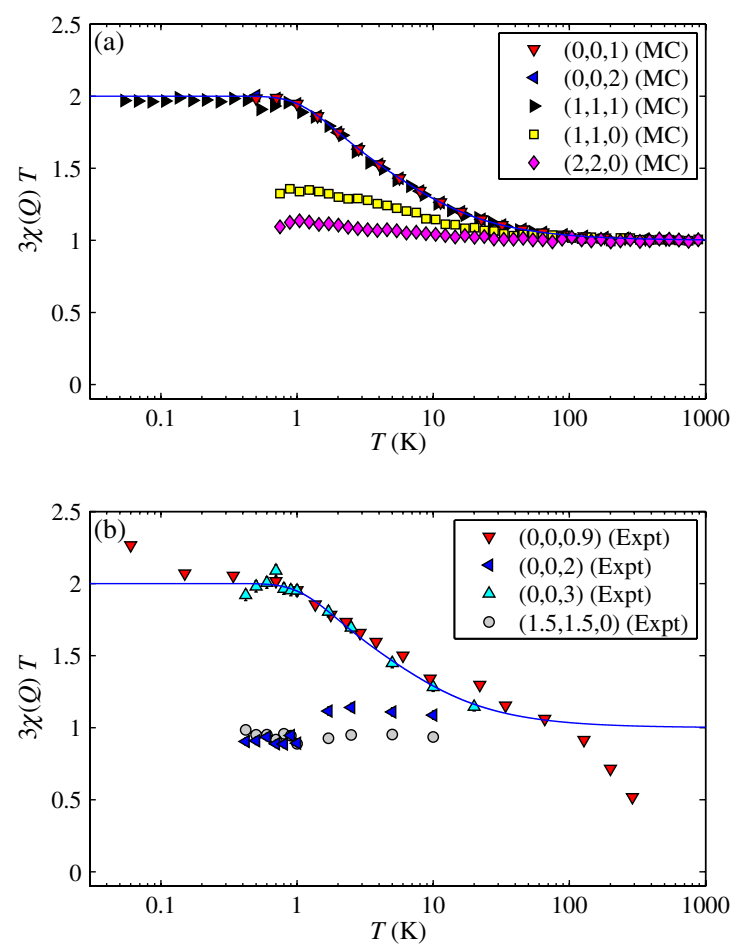

FIG. 6. Wave-vector-dependent susceptibility compared to theory. Panel (a): Simulations of the NNSI model at selected $\mathbf{Q}$ points, obtained from Monte Carlo simulation. Panel (b): Temperature dependence of $S(\mathbf{Q}, T)$ measured for $\mathrm{Ho}_{2} \mathrm{Ti}_{2} \mathrm{O}_{7}$ (see text for details), compared with theory. for scattering at any pinch point, as confirmed for the point at $[1,1,1]$. The data here, extending down to $0.02 \mathrm{~K}$, have been generated using the loop algorithm, while those for $[0,0,1]$ and $[0,0,2]$ come from local, single spin-flip dynamics. For the latter, the system freezes in the $0.6-0.7-\mathrm{K}$ regime, as expected, as the density of magnetic monopoles falls to zero. However, the crossover to the regime of topological-sector fluctuations is completed before ergodicity is lost. Away from the line of high symmetry $[0,0, \ell]$ and from pinch points, the scattering intensity fails to develop as the constraints are imposed but remains larger than the high-temperature asymptote. As seen from the data at $[1,1,0]$ and $[2,2,0]$, the topological-sector information is not contained in these projections, or at least not in a straightforward way.

\section{A. Scattering along the ridge}

The pinch-point function [Eq. (9)] is arranged to give scattering of constant amplitude along [0, 0, $\ell$ [63]. It is perhaps surprising, however, to find that, for the numerical scattering from the NNSI, this long-wavelength expression holds all the way from the zone center (pinch point) to the zone boundary. The $\tilde{\mathbf{q}}=\left(\tilde{q}_{x}, \tilde{q}_{y}, \tilde{q}_{z}\right)$ independence is a consequence of the collective paramagnetism yielding diffuse, rather than either Bragg or critical, scattering. At finite $\tilde{\mathbf{q}}$, away from the zone center along the cubic axis $[0,0, \ell]$, one observes topological-sector fluctuations in an effective system with reduced size of approximately $2 \pi /|\tilde{\mathbf{q}}|$. This effective system has only small finite-size corrections to the thermodynamic limit.

Specifically, for $\mathbf{q}$ along $\hat{z}$, we are interested in fluctuations in $M_{x} \propto w_{x}$ and $M_{y} \propto w_{y}$. The strings of alternating "out-in-out-in ..." spins along a given cubic axis behave as random walkers in the perpendicular plane [64,65]. Thus, those strings oriented along the $\hat{y}$ axis have a ballistic trajectory in this direction but make a diffusive random walk in the $(\hat{x}, \hat{z})$ plane. String correlations are therefore lost when the extension of the string in the $\hat{z}$ direction exceeds $\lambda=2 \pi /|\mathbf{q}|$. If the number of steps along the $\hat{y}$ axis is $\tilde{\ell}_{y}$, the diffusive orthogonal extension in the $(\hat{x}, \hat{z})$ plane is approximately $\sqrt{\tilde{\ell}_{y}}$. For a string oriented along the $\hat{y}$ axis, each tetrahedron provides two possible paths, alternatively, along the $[1,0,1]$ and $[1,0, \overline{1}]$ axes. Hence, such strings make an almost isotropic random-walk step in the $(\hat{x}, \hat{z})$ plane after spanning two tetrahedra. As there are four tetrahedra in a cubic cell, a step length is $a / 2$. Fixing the perpendicular extension as $(a / 2) \sqrt{\tilde{\ell}_{y}}=\lambda$ gives $\tilde{\ell}_{y} \sim$ $(2 \lambda / a)^{2}$, a number that is always greater than unity, even at the zone boundary, where $\lambda=a$ and $\tilde{\ell}_{y}$ spans two cubic cells. Hence, even at the zone boundary, we are observing strings for a system of large enough effective size to be essentially in the asymptotic regime where one can observe topological-sector fluctuations. Although this argument 
relates to a window of finite size embedded in a larger system, rather than to one with periodic boundaries [22], the soft effective boundaries provided by a finite-scattering wave vector appear to give similar results, allowing a constant scattering amplitude along the entire $[0,0, \ell]$ ridge.

In pictorial terms (see Fig. 2), a neutron with momentum transfer $2 \pi / \lambda$ will not detect closed loops on that scale, as such loops do not change the two-point correlation function $g(r=\lambda)$, or the magnetic moment calculated over an area incorporating the loop. However, such a neutron will be sensitive to fluctuations on a larger scale $(r>\lambda)$, which appear as fluctuations of strings as a result of the anisotropic scaling of the string trajectory. Hence, the strings should give essentially uniform scattering right up to the zone boundary.

For a detailed theoretical discussion of the pinch point physics, see the recent paper by Sen et al. [66].

\section{NEUTRON SCATTERING EXPERIMENTS $\mathrm{ON} \mathrm{Ho}_{2} \mathrm{Ti}_{2} \mathrm{O}_{7}$}

We now compare our theoretical and numerical findings with experimental measurements of $S(\mathbf{Q})$. In Fig. 6(b), we show $S(\mathbf{Q})$ for several $\mathbf{Q}$ values in the $[h, h, \ell]$ plane from single-crystal neutron-scattering experiments of $\mathrm{Ho}_{2} \mathrm{Ti}_{2} \mathrm{O}_{7}$. (See the Appendix for details.) For each data set, the intensity is scaled to the value of the susceptibility estimated from the Husimi cactus at a single temperature between 30 and $100 \mathrm{~K}$. There are no fitting parameters for the temperature axis. One can immediately see that the total intensity at or near the zone boundaries $[0,0,0.9]$ and $[0,0,3]$ is in remarkably good agreement with theory and simulation and shows the clear signature of a crossover between paramagnetic fluctuations and topological-sector fluctuations. The departure from the theory at high temperatures is most likely due to increasing thermal population of higher crystal-field levels leading to a loss of elastic spectral weight, an effect that cannot be captured by the ideal Ising spins of the theory.

However, the situation is very different at the pinch point $[0,0,2]$. After an initial increase above the Curie law below $30 \mathrm{~K}$, the value of the scattering intensity stagnates and even decreases as the temperature dips below $3 \mathrm{~K}$. Even though experiment does not follow the NNSI, the pinch points become sharply developed at low temperatures, indicating that the topological constraints are imposed to an excellent approximation [29]. We also show the evolution of the scattering intensity at points along the $[h, h, 0]$ axis. The data for $h=3 / 2$ are similar to the numerical scattering data from the NNSI when scaled to the theoretical susceptibility at high temperatures. As the temperature is reduced, the intensity increases slightly before approaching a plateau, well below the theoretical predictions for scattering along the cubic axis $[0,0,1]$. This result is to be expected, as the spin components perpendicular to this low-symmetry direction fail to capture the correlations of the Coulomb phase.

The differences in behavior at the zone center and at the zone boundaries shows that in $\mathrm{Ho}_{2} \mathrm{Ti}_{2} \mathrm{O}_{7}$, unlike in the NNSI model, not all Pauling states have the same weight. This observation suggests that states with closed loops of flippable spins on many length scales, which are characterized by strong pinch-point scattering, have a lower weight than states characterized by short loops only, which have enhanced scattering intensity at the zone boundaries. Such a variation is consistent with the phenomenology of Youngblood and Axe [63], whose free-energy functional allows for the variation in scattering amplitude along the $[0,0, \ell]$ ridge. The variation is apparent in both Ref. [29], where polarized neutron scattering was required to expose the pinch points, and Ref. [67], where unpolarized scattering in the related spin-ice material $\mathrm{Dy}_{2} \mathrm{Ti}_{2} \mathrm{O}_{7}$ tends toward a pattern characteristic of closed six-spin loops, leaving pinch points of much-reduced amplitude. The long-range nature of the dipolar interactions could be at the origin of the varying amplitude. In the dipolar spin-ice model, the degeneracy of the Pauling states is weakly lifted, although the ensuing phase transition [68] is not observed experimentally. Further neighbor-exchange coupling has also been evoked to explain states dominated by six-spin loops [67]. More exotically, it has also recently been shown $[13,14,69,70]$ that quantum fluctuations can favor states with only short loops and that the inclusion of quantum fluctuations over short loops in a nearest-neighbor spin-ice model can produce simulated neutron-scattering patterns that look remarkably similar to those for $\mathrm{Ho}_{2} \mathrm{Ti}_{2} \mathrm{O}_{7}$ [29].

The intensity variation along the $[0,0, \ell]$ ridge is further investigated in Fig. 7, where we replot data from Ref. [29]. In part (a), we show data at a sequence of points on $[0,0, \ell]$ as a function of temperature. In each case, the scattering intensity is scaled to the predicted susceptibility at $20 \mathrm{~K}$. A continuous evolution away from the theoretical curve is visible between $[0,0,3]$ (the zone boundary) and $[0,0,2]$ (the zone center). In part (b), we show the evolution of scattering intensity at $T=1.7 \mathrm{~K}$ for all $Q$ points along the ridge and compare this evolution with the theoretical expectation at this temperature. The points are folded into a single Brillouin zone. The falloff in scattering intensity along the ridge is approximately linear, with perhaps a hint of curvature toward a constant value above $\ell=0.8$. The linear evolution of intensities between the values we have developed for a paramagnetic and those for a spin-liquid Curie law would indicate that there is, in fact, no characteristic scale above which $S(\mathbf{Q})$, and hence the magnetic susceptibility, returns to the paramagnetic regime. A tentative conclusion, compatible with the observation of pinch points, is therefore that topological-sector fluctuations are maintained, although with reduced amplitude, up to a macroscopic length scale. A word of caution, however: $1.7 \mathrm{~K}$ is above the temperature at which the susceptibility 

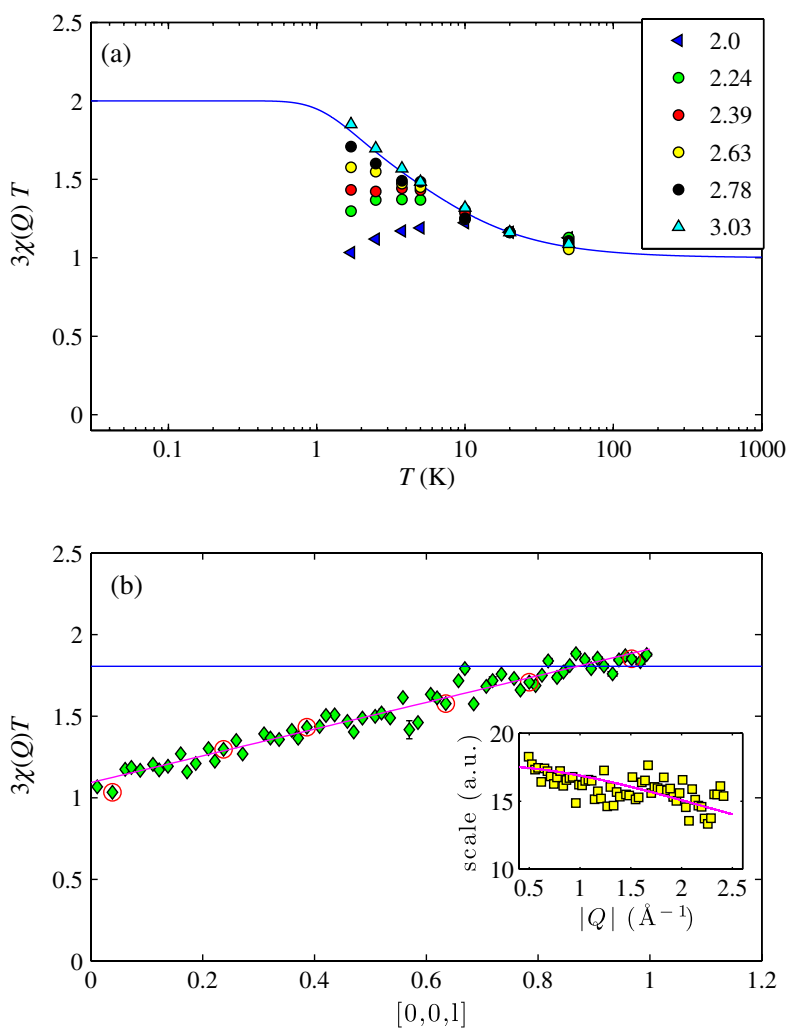

FIG. 7. Wave-vector dependence along the $[0,0, \ell]$ ridge. Panel (a): Temperature dependence of $S(\mathbf{Q}, T)$ at points on $[0,0, \ell]$ scaled to the predicted susceptibility at $20 \mathrm{~K}$. Panel (b): $S(\mathbf{Q}, T)$ vs $(\ell-2)=(a / 2 \pi)|\mathbf{Q}|$ centered at $[0,0,2]$ at $1.7 \mathrm{~K}$. Blue line: Theoretical value at this temperature, from Eq. (1); the pink line in (b) is a guide to the eye. All data points along $[0,0, \ell]$ are folded into a single Brillouin zone, and those ringed in red correspond to the temperature series in panel (a). The inset shows the scale factor for individual points on $[0,0, \ell]$ compared to the squared magnetic form factor $|f(\mathbf{Q})|^{2}$ as a function of $\mathbf{Q}$ at $20 \mathrm{~K}$. (See text for details.)

reaches the asymptotic value characteristic of TSF in the NNSI. For the model system, and presumably for other systems with short-range interactions, we are able to extrapolate to this regime and make clear statements concerning the topological sectors. A full investigation of whether this argument is true in the dipolar spin-ice model would require extensive numerical simulation, which is beyond the scope of the present work.

As, strictly speaking, the excitation of monopoles destroys the Coulomb phase, one might consider the $[0,0, \ell]$ ridge intensity to be modulated by a characteristic scale related to the monopole separation. At $1.7 \mathrm{~K}$, this length scale is now estimated to be $\xi_{m} \sim 15 \AA$ [71], giving a characteristic wave vector of $2 \pi / \xi_{m} \sim 0.68$, in rough agreement with the $\ell=0.8$ estimated above. However, we do not believe the $[0,0, \ell]$ ridge to be modulated in this way, as the monopole separation, which controls the pinch-point width, evolves more rapidly with temperature than does the ridge modulation (consistent with the phenomenology of Youngblood and Axe [63] when translated to magnetism). More measurements are needed to examine this point in detail, but it is worth noting here that the absence of any monopole-related length scale for ridge modulation is consistent with the observed robustness of $S(\mathbf{Q})$ to a small but finite concentration of monopoles. In the inset of Fig. 7(b), we show the $|\mathbf{Q}|$ dependence of the scattering at $20 \mathrm{~K}$ compared to the (scaled) magnetic form factor of $\mathrm{Ho}^{3+}$ (pink line). The 20-K scattering was used as the scale factor for the comparison between theory and experiment at lower temperatures, which therefore removed the form-factor dependence from the experimental data.

Loss of ergodicity could also be responsible for the observed differences between experiment and theory, but we do not believe that to be the case. In all instances, as we have already discussed for numerical simulation, the fate of the comparison between experiment and theory is determined at a temperature above the characteristic $T \sim 0.6 \mathrm{~K}$, where a splitting occurs between field-cooled and zero-field-cooled susceptibilities [72]. This loss of ergodicity is driven by the rarefaction of topological defects, which hinders magnetic relaxation both in numerical simulations and in experiment $[52,72-76]$ and will ultimately lead to ergodicity breaking between topological sectors [53]. However, the crossover to the low-temperature spin-liquid susceptibility is completed before ergodicity is lost, allowing for an extrapolation into the Coulomb phase with associated topologicalsector fluctuations.

Finally for this section, we remark that the bulksusceptibility measurements are in close agreement with the predictions of the NNSI, as regards the Curie-law crossover (Figs. 4 and 6). At first sight, this result is surprising, given that the susceptibility estimated by neutron scattering at the Brillouin-zone center $(0,0,2)$ does not show the full crossover. To explain this difference, one should consider, among other things, that the neutron scattering, even at the zone center, probes spin correlations over much smaller length scales than the bulk measurement. The applied field for the bulk measurement is corrected for the spatial truncation of the magnetic dipole-dipole interaction by using a standard demagnetizing factor, while agreement between neutron scattering and bulk measurements occurs through the fluctuationdissipation relation. Tracking down the precise cause of the difference defines an intriguing problem for future consideration.

\section{TOPOLOGICAL SECTORS IN OTHER SYSTEMS}

Finally, we address the question of generality, as we have argued that the enhanced fluctuations occurring in spin ice at low temperatures provide an implicit measure of emergent gauge phenomena and consequent fluctuations 
in the topological sector of the gauge constraint. Similar emergent phenomena have recently been reported in classical dimer systems [77] and in magnetic pyrochlore, kagome, and checkerboard systems [22-26,78]. In spinice models and materials, the gauge field is proportional to the coarse-grained field of magnetic moments. Hence, it is directly accessible through both bulk-measurement and scattering experiments. This direct access to the gauge field [29] makes spin ice of particular interest in this context, as we have shown here. In pyrochlore antiferromagnets, the gauge field is a hidden property of the rule of satisfied units [79] and essentially corresponds to an inverse mapping between antiferromagnetically coupled spins and ferromagnetically coupled pseudospins. The latter resemble the spin-ice degrees of freedom and form an effective magnetic field within a coarse-grained description [24]. Hence, the physics of the emergent gauge field is common to both classes of systems. For the Heisenberg antiferromagnet, within a Gaussian approximation, the susceptibility for the pseudospin degrees of freedom is enhanced by the same factor of 2 as the susceptibility in spin ice, as the system is cooled into the Coulomb phase, and is related to the stiffness of the Coulomb phase flux field [26]. Although the pseudospin degrees of freedom are not accessible through bulk measurement, their presence appears in magnetic scattering in the form of $S(\mathbf{Q})$ maps with pinch points of differently detailed form from those for spin ice but encoding equivalent information. For nearest-neighbor models, one finds lines of constant scattering, this time along the $[h, h, 0]$ axes. As further neighbor interactions are added, the intensity at the Brillouinzone center is found to dip [26], even though the lowest energy states remain within the topologically constrained phase space. This result seems in complete analogy with our experimental observations along the $[0,0, \ell]$ axis in spin ice, giving weight to our interpretation in terms of topological-sector fluctuations of varying intensity, as the effective length scale, $\ell \sim 1 /|\mathbf{Q}|$, changes from the zone center to the zone boundary.

\section{CONCLUSION}

The local divergence-free constraint of the Coulomb phase in spin-ice materials allows a decomposition of the ground-state ensemble in terms of topological sectors. Fluctuations between sectors are clearly visible through susceptibility measurements, as the winding numbers characterizing the sectors are directly proportional to the magnetization. This is true only in the Coulomb phase, and, as the system settles into that phase, dipolar spin correlations develop, giving a Curie-law crossover between paramagnetism and fluctuations characteristic of the topological spin-liquid phase. The scale factor between the two Curie laws is exactly 2 for our nearest-neighbor model on a Husimi cactus and 2 to a very close approximation for the NNSI model on a pyrochlore lattice. For other systems, it may take different values, but the crossover itself appears quite general and, together with the observation of pinch points, can be taken as an indicator of topological-sector fluctuations.

We have compared analytical and numerical results for the nearest-neighbor spin-ice model with bulkmagnetometry measurements and neutron-scattering measurements through the structure function $S(\mathbf{Q}, T)$ for a single crystal of $\mathrm{Ho}_{2} \mathrm{Ti}_{2} \mathrm{O}_{7}$. We find quantitative agreement between theory and experiment for bulk magnetometry and for scattering at large $\mathbf{Q}$, but near the pinch point, at small wave vectors, the experimental scattering intensity is suppressed compared with theory. We show, however, that the amplitude suppression is not characterized by a single length scale, even though the mean monopole separation scale is in the center of the range of scales probed by neutron scattering at low temperatures. More experimental and theoretical work is required to understand these mechanisms in detail.

We believe this Curie-law crossover is, in fact, a general feature of many frustrated systems [56], apparent as TSF in spin-ice materials and encoded into the scattering pattern of related antiferromagnets. As a consequence, the standard Curie-Weiss picture at high temperatures appears to be incomplete and should be used with caution. Further work in this direction, and, in particular, in potential quantum spin-liquid compounds-herbertsmithite [80], $\mathrm{Tb}_{2} \mathrm{Ti}_{2} \mathrm{O}_{7}$ [81-83], $\mathrm{Yb}_{2} \mathrm{Ti}_{2} \mathrm{O}_{7}[12,84]$ —would be particularly interesting.

It is remarkable that, in spin ice, a completely local probe readily accessible by experiment is able to identify fluctuations between topological sectors, i.e., the difference between "topological constraints" and "topological order." Until now, this task necessarily fell to nonlocal probes, such as measures of the winding number, or the topological-entanglement entropy $[18,19]$. In gapless $U(1)$ quantum liquids, the notion of topological-entanglement entropy is expected to be ill defined [85]. In light of the revelation that there may be other, as yet undiscovered topological invariants in related systems [20,21], it is interesting to speculate whether similar probes may prove instrumental in characterizing this important class of topological order in the future.

\section{ACKNOWLEDGMENTS}

It is a pleasure to thank F. Begue, M. Gingras, A. Harman-Clarke, M. Hastings, L.-P. Henry, Z. Hiroi, G. Misguich, R. Moessner, T. Roscilde, and N. Shannon for useful discussions; A. R. Wildes and M. Boehm for contributions to the IN14 experiments; O. Losserand for cryogenic support during the second experiment; and D. Prabhakaran for a sample. We are grateful for financial support from the European Science Foundation PESC/ RNP/HFM (L.D.C.J. and P.C.W.H.), the Ecole 
Normale Supérieure de Lyon (R. G. M.), and the Institut Universitaire de France (P.C. W. H.).

\section{APPENDIX: DETAILS OF NEUTRON- SCATTERING EXPERIMENTS}

Neutron-scattering data from three different experiments have been presented. In Fig. 6(b), the temperature dependence of the intensity at $[0,0,0.9]$ was obtained using a large flux-grown single crystal of $\mathrm{Ho}_{2} \mathrm{Ti}_{2} \mathrm{O}_{7}$ and IN14 triple-axis spectrometer at the the Institut Laue-Langevin. The IN14 was operated with a graphite monochromator and analyzer. Detailed energy scans of the magnetic scattering showed that it is quasielastic, with an energy width below $0.1 \mathrm{meV}$ for temperatures below about $200 \mathrm{~K}$. We found that, for the temperature scale of interest $(0.1-100 \mathrm{~K})$, our measurements integrated all magnetic fluctuations in the relevant energy window and provided a good measure of $S(\mathbf{Q})$. This sample and experimental configuration were also reported in Ref. [86].

The other reciprocal space positions were investigated in a second experiment using a large crystal of $\mathrm{Ho}_{2} \mathrm{Ti}_{2} \mathrm{O}_{7}$ grown in an image furnace. In this experiment, IN14 was operated in polarized mode, with a Heusler analyzer, giving a similar energy resolution. The reported intensities were obtained by combining measurements of spin-flip and non-spin-flip scattering with vertical incident polarization. Further results of this experiment are as yet unpublished.

The data discussed in Fig. 7 were obtained using the D7 diffuse-scattering spectrometer at the ILL and the second sample mentioned above. These data were extracted from a series of reciprocal space maps, some of which were originally presented in Ref. [29], again combining the spin-flip and non-spin-flip cross sections. In this case, scattering was integrated up to the incident energy $(3.5 \mathrm{meV})$. It is known that there is no significant inelastic scattering in $\mathrm{Ho}_{2} \mathrm{Ti}_{2} \mathrm{O}_{7}$ in this temperature range [87], and the very small quasielastic broadening [61] would have also been integrated in the triple-axis experiments. Thus, all three experiments correspond equally well to a measurement of $S(\mathbf{Q})$.

[1] A.P. Ramirez, Quantum Spin Liquids: A Flood or a Trickle?, Nat. Phys. 4, 442 (2008).

[2] P. A. Lee, An End to the Drought of Quantum Spin Liquids, Science 321, 1306 (2008).

[3] L. Savary and L. Balents, Coulombic Quantum Liquids in Spin-1/2 Pyrochlores, Phys. Rev. Lett. 108, 037202 (2012).

[4] J.D. Thompson, P. A. McClarty, H. M. Ronnow, L.P. Regnault, A. Sorge, and M.J.P. Gingras, Rods of Neutron Scattering Intensity in $\mathrm{Yb}_{2} \mathrm{Ti}_{2} \mathrm{O}_{7}$ : Compelling Evidence for Significant Anisotropic Exchange in a Magnetic Pyrochlore Oxide, Phys. Rev. Lett. 106, 187202 (2011).
[5] L.-J. Chang, S. Onoda, Y. Su, Y.-J. Kao, K.-D. Tsuei, Y. Yasui, K. Kakurai, and M. R. Lees, Higgs Transition from a Magnetic Coulomb Liquid to a Ferromagnet in $\mathrm{Yb}_{2} \mathrm{Ti}_{2} \mathrm{O}_{7}$, Nat. Commun. 3, 992 (2012).

[6] R. Applegate, N. Hayre, R. Singh, T. Lin, A. Day, and M. Gingras, Vindication of $\mathrm{Yb}_{2} \mathrm{Ti}_{2} \mathrm{O}_{7}$ as a Model Exchange Quantum Spin Ice, Phys. Rev. Lett. 109, 097205 (2012).

[7] E. Kermarrec, P. Mendels, F. Bert, R. H. Colman, A. S. Wills, P. Strobel, P. Bonville, A. Hillier, and A. Amato, Spin-Liquid Ground State in the Frustrated Kagome Antiferromagnet $\mathrm{MgCu}_{3}(\mathrm{OH})_{6} \mathrm{Cl}_{2}$, Phys. Rev. B 84, 100401 (2011).

[8] B. Fåk, E. Kermarrec, L. Messio, B. Bernu, C. Lhuillier, F. Bert, P. Mendels, B. Koteswararao, F. Bouquet, J. Ollivier et al., Kapellasite: A Kagome Quantum Spin Liquid with Competing Interactions, Phys. Rev. Lett. 109, 037208 (2012).

[9] L. Messio, B. Bernu, and C. Lhuillier, Kagome Antiferromagnet: A Chiral Topological Spin Liquid?, Phys. Rev. Lett. 108, 207204 (2012).

[10] T. Han, S. Chu, and Y.S. Lee, Refining the Spin Hamiltonian in the Spin- $\frac{1}{2}$ Kagome Lattice Antiferromagnet $\mathrm{ZnCu}_{3}(\mathrm{OH})_{6} \mathrm{Cl}_{2}$ Using Single Crystals, Phys. Rev. Lett. 108, 157202 (2012).

[11] M. Hermele, M. P. A. Fisher, and L. Balents, Pyrochlore Photons: The U(1) Spin Liquid in a $S=1 / 2$ ThreeDimensional Frustrated Magnet, Phys. Rev. B 69, 064404 (2004).

[12] K. Ross, L. Savary, B. D. Gaulin, and L. Balents, Quantum Excitations in Quantum Spin Ice, Phys. Rev. X 1, 21002 (2011).

[13] O. Benton, O. Sikora, and N. Shannon, Seeing the Light: Experimental Signatures of Emergent Electromagnetism in a Quantum Spin Ice, Phys. Rev. B 86, 075154 (2012).

[14] N. Shannon, O. Sikora, F. Pollmann, K. Penc, and P. Fulde, Quantum Ice: A Quantum Monte Carlo Study, Phys. Rev. Lett. 108, 067204 (2012).

[15] L. Balents, Spin Liquids in Frustrated Magnets, Nature (London) 464, 199 (2010).

[16] C. Castelnovo, R. Moessner, S. L. Sondhi, Spin Ice, Fractionalization, and Topological Order, Annu. Rev. Condens. Matter Phys. 3, 35 (2012).

[17] X. G. Wen, Quantum Orders and Symmetric Spin Liquids, Phys. Rev. B 65, 165113 (2002).

[18] M. Levin and X. G. Wen, Detecting Topological Order in a Ground State Wave Function, Phys. Rev. Lett. 96, 110405 (2006).

[19] A. Kitaev and J. Preskill, Topological Entanglement Entropy, Phys. Rev. Lett. 96, 110404 (2006).

[20] Y. Ran, P. Hosur, and A. Vishwanath, Fermionic Hopf Solitons and Berry Phase in Topological Surface Superconductors, Phys. Rev. B 84, 184501 (2011).

[21] M. Freedman, M. B. Hastings, C. Nayak, and X.-L. Qi, Weakly Coupled Non-Abelian Anyons in Three Dimensions, Phys. Rev. B 84, 245119 (2011).

[22] A. J. Macdonald, P. C. W. Holdsworth, and R. G. Melko, Classical Topological Order in Kagome Ice, J. Phys. Condens. Matter 23, 164208 (2011).

[23] B. Canals and D. A. Garanin, Spin-Liquid Phase in the Pyrochlore Anti-ferromagnet, Can. J. Phys. 79, 1323 (2001). 
[24] S. V. Isakov, K. Gregor, R. Moessner, and S. L. Sondhi, Dipolar Spin Correlations in Classical Pyrochlore Magnets, Phys. Rev. Lett. 93, 167204 (2004).

[25] C. L. Henley, Power-Law Spin Correlations in Pyrochlore Antiferromagnets, Phys. Rev. B 71, 014424 (2005).

[26] P. H. Conlon and J. T. Chalker, Absent Pinch Points and Emergent Clusters: Further Neighbor Interactions in the Pyrochlore Heisenberg Antiferromagnet, Phys. Rev. B 81, 237206 (2010).

[27] M.P. Zinkin, M.J. Harris, and T. Zeiske, Short-Range Magnetic Order in the Frustrated Pyrochlore Antiferromagnet $\mathrm{CsNiCrF}_{6}$, Phys. Rev. B 56, 11786 (1997).

[28] T. Fennell, S. T. Bramwell, D. F. McMorrow, P. Manuel, and A. R. Wildes, Pinch Points and Kasteleyn Transitions in Kagome Ice, Nat. Phys. 3, 566 (2007).

[29] T. Fennell, P.P. Deen, A. R. Wildes, K. Schmalzl, D. Prabhakaran, A. T. Boothroyd, R.J. Aldus, D.F. McMorrow, and S.T. Bramwell, Magnetic Coulomb Phase in the Spin Ice $\mathrm{Ho}_{2} \mathrm{Ti}_{2} \mathrm{O}_{7}$, Science 326, 415 (2009).

[30] C.L. Henley, The "Coulomb Phase" in Frustrated Systems, Annu. Rev. Condens. Matter Phys. 1, 179 (2010).

[31] S. T. Bramwell and M. J. Harris, Frustration in Ising-Type Spin Models on the Pyrochlore Lattice, J. Phys. Condens. Matter 10, L215 (1998).

[32] H. Kadowaki, N. Doi, Y. Aoki, Y. Tabata, T. J. Sato, J. W. Lynn, K. Matsuhira, and Z. Hiroi, Observation of Magnetic Monopoles in Spin Ice, J. Phys. Soc. Jpn. 78, 103706 (2009).

[33] L. J. Chang, Y. Su, Y.-J. Kao, Y.Z. Chou, R. Mittal, H. Schneider, T. Brückel, G. Balakrishnan, and M. R. Lees, Magnetic Correlations in the Spin Ice $\mathrm{Ho}_{2-x} \mathrm{Y}_{x} \mathrm{Ti}_{2} \mathrm{O}_{7}$ as Revealed by Neutron Polarization Analysis, Phys. Rev. B 82, 172403 (2010).

[34] S. T. Bramwell, M. J. Harris, B.C. den Hertog, M. J. P. Gingras, J. S. Gardner, D. F. McMorrow, A.R. Wildes, A. L. Cornelius, J. D. M. Champion, R. G. Melko et al., Spin Correlations in $\mathrm{Ho}_{2} \mathrm{Ti}_{2} \mathrm{O}_{7}:$ A Dipolar Spin Ice System, Phys. Rev. Lett. 87, 047205 (2001).

[35] M. J. Harris and S. T. Bramwell (unpublished).

[36] P.W. Anderson, Ordering and Antiferromagnetism in Ferrites, Phys. Rev. 102, 1008 (1956).

[37] R. Moessner, Relief and Generation of Frustration in Pyrochlore Magnets by Single-Ion Anisotropy, Phys. Rev. B 57, R5587 (1998).

[38] L. Pauling, The Structure and Entropy of Ice and of Other Crystals with Some Randomness of Atomic Arrangement, J. Am. Chem. Soc. 57, 2680 (1935).

[39] A. P. Ramirez, A. Hayashi, R. J. Cava, R. Siddharthan, and B. S. Shastry, Zero-Point Entropy in "Spin Ice", Nature (London) 399, 333 (1999).

[40] H. Cao, A. Gukasov, I. Mirebeau, P. Bonville, C. Decorse, and G. Dhalenne, Ising Versus XY Anisotropy in Frustrated $\mathrm{R}_{2} \mathrm{Ti}_{2} \mathrm{O}_{7}$ Compounds as Seen by Polarized Neutrons, Phys. Rev. Lett. 103, 056402 (2009).

[41] L. D. C. Jaubert, J. T. Chalker, P. C. W. Holdsworth, and R. Moessner, Three-Dimensional Kasteleyn Transition: Spin Ice in a [100] Field, Phys. Rev. Lett. 100, 067207 (2008).
[42] S. Powell and J. T. Chalker, Classical to Quantum Mappings for Geometrically Frustrated Systems: SpinIce in a [100] Field, Phys. Rev. B 78, 024422 (2008).

[43] S. Powell, Higgs Transitions of Spin Ice, Phys. Rev. B 84, 094437 (2011).

[44] S. V. Isakov, K. S. Raman, R. Moessner, and S. L. Sondhi, Magnetization Curve of Spin Ice in a [111] Magnetic Field, Phys. Rev. B 70, 104418 (2004).

[45] Also called a loop algorithm by Melko et al. [46].

[46] R. G. Melko and M. J. P. Gingras, Monte Carlo Studies of the Dipolar Spin Ice Model, J. Phys. Condens. Matter 16, R1277 (2004).

[47] R. Moessner and S.L. Sondhi, Theory of the [111] Magnetization Plateau in Spin Ice, Phys. Rev. B 68, 064411 (2003).

[48] G. I. Watson, Symmetry Relations for the Six-Vertex Model, J. Stat. Phys. 94, 1045 (1999).

[49] D. J. P. Morris, D. A. Tennant, S. A. Grigera, B. Klemke, C. Castelnovo, R. Moessner, C. Czternasty, M. Meissner, K. C. Rule, J. U. Hoffmann et al., Dirac Strings and Magnetic Monopoles in the Spin Ice $\mathrm{Dy}_{2} \mathrm{Ti}_{2} \mathrm{O}_{7}$, Science 326, 411 (2009).

[50] C. Castelnovo, R. Moessner, and S. L. Sondhi, Magnetic Monopoles in Spin Ice, Nature (London) 451, 42 (2008).

[51] I. A. Ryzhkin, Magnetic Relaxation in Rare-Earth Oxide Pyrochlores, J. Exp. Theor. Phys. 101, 481 (2005).

[52] L.D.C. Jaubert and P.C. W. Holdsworth, Signature of Magnetic Monopole and Dirac String Dynamics in Spin Ice, Nat. Phys. 5, 258 (2009).

[53] C. Castelnovo and C. Chamon, Topological Order and Topological Entropy in Classical Systems, Phys. Rev. B 76, 174416 (2007).

[54] S. Yoshida, K. Nemoto, and K. Wada, Application of the Cluster Variation Method to Spin Ice Systems on the Pyrochlore Lattice, J. Phys. Soc. Jpn. 71, 948 (2002).

[55] L. D. C. Jaubert, Topological Constraints and Defects in Spin Ice, Ph.D. thesis, Ecole Normale Supérieure de Lyon, 2009.

[56] L.D.C. Jaubert and P.C.W. Holdsworth, Curie Law Crossover in Frustrated Systems (unpublished).

[57] B.C. den Hertog and M. J.P. Gingras, Dipolar Interactions and Origin of Spin Ice in Ising Pyrochlore Magnets, Phys. Rev. Lett. 84, 3430 (2000).

[58] S. V. Isakov, R. Moessner, and S. L. Sondhi, Why Spin Ice Obeys the Ice Rules, Phys. Rev. Lett. 95, 217201 (2005).

[59] S. T. Bramwell, M. N. Field, M. J. Harris, and I. P. Parkin, Bulk Magnetization of the Heavy Rare Earth Titanate Pyrochlores-A Series of Model Frustrated Magnets, J. Phys. Condens. Matter 12, 483 (2000).

[60] A.P. Ramirez, Strongly Geometrically Frustrated Magnets, Annu. Rev. Mater. Sci. 24, 453 (1994).

[61] J. P. Clancy, J. P. C. Ruff, S. R. Dunsiger, Y. Zhao, H. A. Dabkowska, J.S. Gardner, Y. Qiu, J. R. D. Copley, T. Jenkins, and B.D. Gaulin, Revisiting Static and Dynamic Spin-Ice Correlations in $\mathrm{Ho}_{2} \mathrm{Ti}_{2} \mathrm{O}_{7}$ with Neutron Scattering, Phys. Rev. B 79, 014408 (2009).

[62] See, for example, Eq. 4.12 in H. Kadowaki, Y. Ishii, K. Matsuhira, and Y. Hinatsu, Neutron Scattering Study of Dipolar Spin Ice $\mathrm{Ho}_{2} \mathrm{Sn}_{2} \mathrm{O}_{7}$ : Frustrated Pyrochlore Magnet, Phys. Rev. B 65, 144421 (2002). 
[63] R.W. Youngblood and J.D. Axe, Polarization Fluctuations in Ferroelectric Models, Phys. Rev. B 23, 232 (1981).

[64] S. M. Bhattacharjee, J. F. Nagle, D. A. Huse, and M.E. Fisher, Critical-Behavior of a Three-Dimensional Dimer Model, J. Stat. Phys. 32, 361 (1983).

[65] L. D. C. Jaubert, M. Haque, and R. Moessner, Analysis of a Fully Packed Loop Model Arising in a Magnetic Coulomb Phase, Phys. Rev. Lett. 107, 177202 (2011).

[66] A. Sen, R. Moessner, and S. L. Sondhi, arXiv:1212.2112.

[67] T. Yavors'kii, T. Fennell, M. J.P. Gingras, and S. T. Bramwell, $\mathrm{Dy}_{2} \mathrm{Ti}_{2} \mathrm{O}_{7}$ Spin Ice: A Test Case for Emergent Clusters in a Frustrated Magnet, Phys. Rev. Lett. 101, 037204 (2008).

[68] R. G. Melko, B. C. den Hertog, and M. J. P. Gingras, LongRange Order at Low Temperatures in Dipolar Spin Ice, Phys. Rev. Lett. 87, 067203 (2001).

[69] O. Sikora, F. Pollmann, N. Shannon, K. Penc, and P. Fulde, Quantum Liquid with Deconfined Fractional Excitations in Three Dimensions, Phys. Rev. Lett. 103, 247001 (2009).

[70] O. Sikora, N. Shannon, F. Pollmann, K. Penc, and P. Fulde, Extended Quantum U(1)-Liquid Phase in a ThreeDimensional Quantum Dimer Model, Phys. Rev. B 84, 115129 (2011).

[71] T. Fennell et al. (unpublished).

[72] J. Snyder, B. G. Ueland, J. S. Slusky, H. Karunadasa, R. J. Cava, and P. Schiffer, Low-Temperature Spin Freezing in the $\mathrm{Dy}_{2} \mathrm{Ti}_{2} \mathrm{O}_{7}$ Spin Ice, Phys. Rev. B 69, 064414 (2004).

[73] K. Matsuhira, Y. Hinatsu, K. Tenya, and T. Sakakibara, Low Temperature Magnetic Properties of Frustrated Pyrochlore Ferromagnets $\mathrm{Ho}_{2} \mathrm{Sn}_{2} \mathrm{O}_{7}$ and $\mathrm{Ho}_{2} \mathrm{Ti}_{2} \mathrm{O}_{7}$, J. Phys. Condens. Matter 12, L649 (2000).

[74] E. Lhotel, C. Paulsen, P. Dalmas de Réotier, A. Yaouanc, C. Marin, and S. Vanishri, Low-Temperature Magnetization in Geometrically Frustrated $\mathrm{Tb}_{2} \mathrm{Ti}_{2} \mathrm{O}_{7}$, Phys. Rev. B 86, 020410 (2012).

[75] G. Ehlers, A. L. Cornelius, M. Orendac, M. Kajnakova, T. Fennell, S. T. Bramwell, and J.S. Gardner, Dynamical Crossover in 'Hot' Spin Ice, J. Phys. Condens. Matter 15, L9 (2003).

[76] L. R. Yaraskavitch, H. M. Revell, S. Meng, K. A. Ross, H. M. L. Noad, H. A. Dabkowska, B. D. Gaulin, and J. B. Kycia, Spin Dynamics in the Frozen State of the Dipolar
Spin Ice Material $\mathrm{Dy}_{2} \mathrm{Ti}_{2} \mathrm{O}_{7}$, Phys. Rev. B 85, 020410 (2012).

[77] D. A. Huse, W. Krauth, R. Moessner, and S. L. Sondhi, Coulomb and Liquid Dimer Models in Three Dimensions, Phys. Rev. Lett. 91, 167004 (2003).

[78] G. Misguich, B. Bernu, and L. Pierre, Determination of the Exchange Energies in $\mathrm{Li}_{2} \mathrm{VOSiO}_{4}$ from a HighTemperature Series Analysis of the Square-Lattice J(1)-J(2) Heisenberg Model, Phys. Rev. B 68, 113409 (2003).

[79] R. Moessner, Magnets with Strong Geometric Frustration, Can. J. Phys. 79, 1283 (2001).

[80] P. Mendels, F. Bert, M. A. de Vries, A. Olariu, A. Harrison, F. Duc, J.C. Trombe, J.S. Lord, A. Amato, and C. Baines, Quantum Magnetism in the Paratacamite Family: Towards an Ideal Kagome Lattice, Phys. Rev. Lett. 98, 077204 (2007).

[81] J. S. Gardner, A. Keren, G. Ehlers, C. Stock, E. Segal, J. M. Roper, B. Fak, M. B. Stone, P. R. Hammar, D. H. Reich et al., Dynamic Frustrated Magnetism in $\mathrm{Tb}_{2} \mathrm{Ti}_{2} \mathrm{O}_{7}$ at $50 \mathrm{mK}$, Phys. Rev. B 68, 180401 (2003).

[82] J.S. Gardner, M. J.P. Gingras, and J.E. Greedan, Magnetic Pyrochlore Oxides, Rev. Mod. Phys. 82, 53 (2010).

[83] T. Fennell, M. Kenzelmann, B. Roessli, M. K. Haas, and R.J. Cava, Power-Law Spin Correlations in the Pyrochlore Antiferromagnet $\mathrm{Tb}_{2} \mathrm{Ti}_{2} \mathrm{O}_{7}$, Phys. Rev. Lett. 109, 017201 (2012).

[84] J. D. Thompson, P. A. McClarty, and M. J.P. Gingras, Local Susceptibility of the $\mathrm{Yb}_{2} \mathrm{Ti}_{2} \mathrm{O}_{7}$ Rare Earth Pyrochlore Computed from a Hamiltonian with Anisotropic Exchange, J. Phys. Condens. Matter 23, 164219 (2011).

[85] H. Ju, A. B. Kallin, P. Fendley, M. B. Hastings, and R. G. Melko, Entanglement Scaling in Two-Dimensional Gapless Systems, Phys. Rev. B 85, 165121 (2012).

[86] S. T. Bramwell and M. J.P. Gingras, Spin Ice State in Frustrated Magnetic Pyrochlore Materials, Science 294, 1495 (2001).

[87] S. Rosenkranz, A. P. Ramirez, A. Hayashi, R. J. Cava, R. Siddharthan, and B.S. Shastry, Crystal-Field Interaction in the Pyrochlore Magnet $\mathrm{Ho}_{2} \mathrm{Ti}_{2} \mathrm{O}_{7}$, J. Appl. Phys. 87, 5914 (2000). 